\title{
Diseño de una escala ecográfica predictora de malignidad en nódulos tiroideos: Comunicación preliminar
}

\author{
José Miguel Domínguez ${ }^{1,2}$, René Baudrand ${ }^{1,2}$, \\ Eugenio Arteaga ${ }^{1,2}$, Claudia Campusano ${ }^{1,2}$, \\ Gilberto G onzález 1,2, Lorena M osso ${ }^{1,2}$, G abriel Cavada ${ }^{5 a}$, \\ Francisco $\mathrm{Cruz}^{3}$, Javiera Torres ${ }^{4}$, Antonieta Solar ${ }^{4}$, \\ Tatiana Arias'2, Alejandra Pizarro², Marcelo Gómez², \\ Carlos Fardella ${ }^{1,2}$.
}

\section{An ultrasound score to predict the presence of papillary thyroid carcinoma. Preliminary report}

Background: Thyroid nodules are common and associated to a low risk of malignancy. Their clinical assessment usually includes a fine neddle aspiration biopsy (FNAB). Aim: To identify ultrasonographic characteristics associated to papillary thyroid carcinoma (PTC) and generate a score that predicts the risk of PTC. Material and methods: Retrospective review of all fine needle aspiration biopsies of the thyroid performed in a lapse of two years. Biopsies that were conclusive for PTC were selected and compared with an equal amount of randomly selected biopsies that disclosed a benign diagnosis. Results: One hundred twenty two biopsies of a total of 1,498 were conclusive for PTC. Univariate analysis showed associations with PTC for the presence of micro-calcifications (Odds ratio (OR) 49.2: 95\% confidence intervals (CI) 18.7-140.9), solid predominance (OR 25.1; 95\% CI 6-220), hypoechogenicity (OR 23.5, 95\% CI 6.5-122.6), irregular borders (OR 17, 95\% CI 7.2-42.9), lymph node involvement (OR 12.3, 95\% CI 2.7-112), central vascularization (OR 12.2, 95\% CI 4.8-33.3), local invasion and hyperechogenicity (OR 0.2; CI 95\% CI 0.03-0.6). Multivariate analysis disclosed microcalcifications (OR 28.1; CI 95\% 8.9-89), hypoechogenicity (OR 9.4; 95\% CI 1.5-59.5) and irregular borders (OR 4.7; CI 95\% 1.5-15) as the variables independently associated with the presence of PTC. The prevalence of PTC in the presence of the three variables was 97.6\% (Likelihood ratio (LR) 45) and 5.4\% in their absence (LR 0.06). Conclusions: This scale predicts the presence or absence of PTC using simple ultrasound characteristics (Rev Méd Chile 2009; 137: 1031-6).

(Key words: Biopsy, fine-needle; Carcinoma, papillary; Thyroid neoplasms)

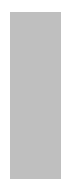

\footnotetext{
${ }^{a}$ Bioestadístico
}

Recibido el 24 de noviembre, 2008. Aceptado el 1 de julio, 2009.

Departamentos de Endocrinología ${ }^{1}$, Centro de Tiroides $^{2}$, Radiología ${ }^{3}$ y Anatomía Patológica ${ }^{4}$, Facultad de Medicina, Pontificia Universidad Católica de Chile. Santiago de Chile. ${ }^{5}$ Escuela de Salud Pública Universidad de Chile, Sociedad Chilena de Endocrinología y Diabetes.

\footnotetext{
$\overline{\text { Correspondencia a: Dr. Carlos E. Fardella. Departamento de }}$ Endocrinología, Facultad de Medicina, Pontificia Universidad Católica de Chile. Lira 85, 5ำ piso. Santiago, Chile. Teléfono: 3543095. Fax: 6385675. E mail: cfardella@med.puc.cl
} 
$\mathrm{L}$ os nódulos tiroideos son una patología prevalente y se encuentran en hasta $70 \%$ de los pacientes que se realizan una ecografía tiroidea ${ }^{1}$. El enfrentamiento habitual incluye la medición de hormona tiroestimulante (TSH) y, en ausencia de hipertiroidismo, el estudio histológico a través de una punción aspirativa con aguja fina (PAAF), cuyo resultado es benigno en $95 \%$ de los casos $^{2}$.

La PAAF tiroidea es un procedimiento económico, rápido y relativamente sencillo. Tiene una sensibilidad entre $65 \%$ y $98 \%$ y una especificidad entre $72 \%$ y 100\% para el diagnóstico de carcinoma papilar tiroideo (CPT) y que ha permitido reducir el número de tiroidectomías innecesarias desde su utilización masiva ${ }^{3}$. Aun así, es una técnica medianamente invasiva, con una tasa de muestras insatisfactorias cercanas a 15\% y que intenta identificar una patología que sólo se encuentra en 5\% de los pacientes que se someten a ella. Por lo anterior, y con el objetivo de optimizar el estudio histológico en pacientes con patología nodular y focalizarlo en aquéllos con mayor riesgo de cáncer, se ha intentado precisar aquellas características ecográficas que permitan estimar, de un modo menos invasivo, el riesgo individual de malignidad en cada paciente ${ }^{3}$.

La ecografía tiroidea permite, además de localizar los nódulos, definir elementos como su tamaño, estructura (sólida o quística), forma (relación altu$\mathrm{ra}$ /ancho), ecogenicidad, características de los bordes, presencia de calcificaciones y halo, patrón predominante del flujo al estudio con doppler y presencia de invasión local o compromiso de linfonodos vecinos ${ }^{3}$. Estos factores han sido evaluados en cuanto a su asociación con carcinoma papilar tiroideo, encontrándose publicaciones con resultados diversos. Al revisar la literatura, descubrimos que los elementos ecográficos asociados a CPT son múltiples y que no existe en la actualidad un consenso sobre cuáles predicen la presencia de neoplasia ni su importancia relativa. Entre los más estudiados destacan la hipoecogenicidad del nódulo, la presencia de microcalcificaciones, bordes irregulares o poco definidos, ausencia de halo hipoecogénico, predominio de flujo central al estudio con Doppler color y la invasión de estructuras vecinas o ganglionares ${ }^{3}$.

Uno de los factores que probablemente influye en la heterogeneidad de los resultados publicados es la ausencia de criterios que estandaricen la forma en que se realiza e interpreta la ecografía. Lo anterior queda de manifiesto al analizar la forma en que se determina la ecogenicidad del tumor, pues mientras algunos autores lo hacen comparando el nódulo con el parénquima tiroideo que lo rodea, otros con los músculos peritiroideos y otros ni siquiera lo especifican.

La evidencia actual permite reconocer que el tamaño de los nódulos no incide en el riesgo de $\mathrm{CPT}^{3,4}$. Asimismo, se han asociado a malignidad la presencia de hipoecogenicidad, microcalcificaciones, bordes irregulares, invasión local, compromiso de uno o más linfonodos y el predominio de un flujo central en el estudio con doppler color; así como la ausencia de un halo que rodee al nódulo $3,5,6$. La asociación de otros elementos como forma y elasticidad del nódulo, son más controversiales como factores de riesgo ${ }^{7-9}$.

El objetivo de este estudio fue identificar las características ecográficas de los nódulos tiroideos asociadas a CPT en biopsias obtenidas por PAAF y, a partir de éstos, desarrollar una escala de puntaje predictora de malignidad.

\section{PaCIENTES y MÉTODO}

Se realizó un análisis de todas las PAAF de nódulos tiroideos realizadas en 1.411 pacientes en el Centro de Tiroides del Departamento de Endocrinología de la Pontificia Universidad Católica de Chile, entre los meses de febrero de 2006 y abril de 2008. Se seleccionaron aquellas concluyentes de CPT y se compararon con igual número de ecografías cuyo estudio histológico por PAAF resultó benigno (tiroiditis linfocitaria o nódulo coloideo), seleccionadas al azar y realizadas en pacientes de género y edad equivalentes. Dada la imposibilidad de precisar su condición de benignidad o malignidad a través de la PAAF, no se incluyeron los nódulos cuyo estudio por este método concluyó lesión folicular.

Tres de los autores (JMD, RB, AP) revisaron de manera retrospectiva, y basados en una pauta preestablecida, los informes de las ecografías realizadas por 6 radiólogos con un ecógrafo Phillips HDX11, utilizando un transductor lineal de alta resolución de 5-12 MHz, y se analizaron de manera oculta al resultado del estudio histológico todos los nódulos. Las características ecográficas 
analizadas y su definición fueron las siguientes: a) Hipo e hiperecogenicidad: presencia de tejido de menor o mayor ecogenicidad respecto al parénquima tiroideo, respectivamente ${ }^{9-12}$. b) Predominio sólido: más del 50\% de la superficie tenía esa consistencia ${ }^{11,13}$. c) Bordes irregulares: imposibilidad de delimitar el nódulo $9,13,14$. d) Microcalcificaciones: presencia de múltiples imágenes hiperecogénicas menores a $1 \mathrm{~mm}$ sin sombra acústica asociada11,12,14. e) Ausencia de halo: imposibilidad de identificar un anillo hipoecogénico alrededor del nódulo ${ }^{13,14}$. f) Vascularización central: predominio de este patrón al estudio con Doppler $\operatorname{color}^{14}$. g) Invasión local: definida por infiltración de estructuras contiguas ${ }^{11}$. h) Adenopatía sospechosa: linfonodo redondeado con microcalcificaciones, hipoecogénico respecto a los músculos peritiroideos, apariencia quística, vascularización difusa o pérdida de hilio ${ }^{12,15}$.

Las PAAF se realizaron bajo guía ecográfica con aguja de 21 gauge. Las muestras obtenidas fueron fijadas en formalina tamponada al $10 \%$. Cada una de ellas se procesó como block, para lo cual fueron centrifugadas a 4.000 revoluciones por minuto durante seis minutos. Posterior a esto se obtuvo un sedimento que fue incluido en parafina $y$ del que se realizaron cortes seriados en al menos dos niveles, de $5 \mu \mathrm{m}$ de espesor y teñidos con hematoxilina eosina. Todos los casos fueron diagnosticados por un patólogo experto de nuestro centro. Se consideraron aptas para diagnóstico histopatológico las muestras que incluyeran al menos seis grupos de quince células evaluables, de acuerdo a lo recomendado por la Organización Mundial de la Salud ${ }^{3}$.

Se estudió la asociación entre el "patrón de oro" y cada característica ecográfica mediante la dócima de independencia basada en la prueba de chi cuadrado o el test de Fisher. Para estimar la fuerza de la asociación se utilizó el Odds Ratio (OR) con su respectivo intervalo de confianza. Mediante un análisis de regresión logística, que incluyó aquellas características que en el análisis univariado resultaron estadísticamente significativas, se creó un modelo predictivo que permitió obtener los coeficientes asociados a cada una de las variables ecográficas analizadas y su relación con la presencia de CPT. El signo del coeficiente indica la dirección en que se mueve la probabilidad y su valor absoluto, el incremento o disminu- ción de riesgo por cada unidad que éste aumente cuando el resto de las variables no se modifica.

Según los coeficientes obtenidos, y con el objetivo que el modelo generado tuviera aplicación clínica, se asignó un puntaje a cada una de las variables incluidas en él, se confeccionó una escala para estimar la probabilidad de presencia o ausencia de CPT según el puntaje obtenido y se calcularon las razones de verosimilitud o likelihood ratio (LR) para presencia de CPT en cada categoría. Se consideró un nivel de significación de 5\% y los intervalos de confianza son de nivel 95\%. Para el análisis estadístico y la generación de la escala de puntaje se utilizó el programa STATA versión 10.0 (StataCorp, Collage Station, TX).

\section{Resultados}

Se realizaron 1.711 PAAF en 1.411 pacientes. Ninguno de los procedimientos se asoció a complicaciones que requirieran de la hospitalización del paciente. Del total de nódulos estudiados, se obtuvo muestra satisfactoria para el estudio histológico en 1.498 (87,5\%), de los cuales $122(8,1 \%)$ correspondieron a CPT y fueron comparados con igual número de punciones con estudio histológico benigno. De los 224 nódulos incluidos en el análisis, $206(89,7 \%)$ provenían de pacientes de género femenino y la edad fue de 46,2 $\pm 14,7$ años. Los nódulos puncionados tuvieron un diámetro de $15,3 \pm 8,8 \mathrm{~mm}$.

En el análisis univariado, se asociaron a CPT la presencia de microcalcificaciones (OR 49,2; IC 95\% 18,7-140,9), predominio sólido (OR 25,1; IC 95\% 6220), hipoecogenicidad (OR 23,5; IC 95\% 6,5122,6), bordes irregulares (OR 17; IC 95\% 7,2-42,9), adenopatía sospechosa (OR 12,3; IC 95\% 2,7-112), vascularización central (OR 12,2; IC 95\% 4,8-33,3), invasión local $(\mathrm{p}<0,05)$ e hiperecogenicidad (OR 0,2; IC 95\% 0,03-0,6) (Tabla 1). El tamaño fue menor en los CPT que en los nódulos benignos $(13,63 \pm 7,9 \mathrm{~mm}$ vs $16,8 \pm 7,9 \mathrm{~mm}, \mathrm{p}=0,005)$.

En la regresión logística mantuvieron la significación: microcalcificaciones (OR 28,1; IC 95\% 8,989), hipoecogenicidad (OR 9,4; IC 95\% 1,5-59,5) y bordes irregulares (OR 4,7; IC 95\% 1,5-15). Este modelo se asoció a una curva ROC con un área de 0,93, lo que refleja su buena capacidad predictiva. Los coeficientes obtenidos para estas variables 


\section{Tabla 1. Resultado del análisis univariado entre características ecográficas y presencia de carcinoma papilar de tiroides (C PT)}

\begin{tabular}{|lcccc|}
\hline Característica ecográfica & CPT & Lesiones benignas & $\mathbf{p}$ & O R (IC 95\%) \\
\hline Microcalcificaciones & $78,6 \%$ & $7 \%$ & $<0,001$ & $49,2(18,7-140,9)$ \\
Predominio sólido & $98 \%$ & $66,7 \%$ & $<0,001$ & $25,1(6-220)$ \\
Hipoecogenicidad & $96,7 \%$ & $55,6 \%$ & $<0,001$ & $23,5(6,8-122,6)$ \\
Bordes irregulares & $60,7 \%$ & $8,3 \%$ & $<0,001$ & $17(7,2-42,9)$ \\
Adenopatía sospechosa & $26 \%$ & $2,8 \%$ & $<0,001$ & $12,3(2,7-112)$ \\
Vascularización central & $60 \%$ & $9 \%$ & $<0,001$ & $12,2(4,8-33,3)$ \\
Invasión local & $7,6 \%$ & $0 \%$ & 0,02 & - \\
Hiperecogenicidad & $3,7 \%$ & $20 \%$ & 0,001 & $0,2(0,03-0,6)$ \\
Ausencia de halo & $66,1 \%$ & $75,3 \%$ & 0,2 & $1,6(0,7-3,5)$ \\
\hline
\end{tabular}

*La ausencia de invasión local en pacientes con patología benigna impidió el cálculo del OR.

con el modelo predictivo aplicado fueron los siguientes: microcalcificaciones 3,3; hipoecogenicidad 2,2 y bordes irregulares 1,35 . En base a ellos, se asignó a estas características ecográficas 3, 2 y 1 punto, respectivamente, y se confeccionó una escala clínicamente aplicable que evaluó la prevalencia de cáncer según la suma total de puntos (Tabla 2). El 24,2\% de los nódulos no presentó ninguna de estas características y sólo 5,4\% de ellos tenía CPT (Likelihood Ratio 0,06). En el otro extremo, 26,8\% de los nódulos estudiados tenía las tres y el 97,6\% de ellos presentaba CPT (Likelihood Ratio 45).

\section{DisCUSIÓN}

Los resultados de este estudio sugieren que la ecotomografía tiroidea y la aplicación de la escala de puntaje generada son capaces de diferenciar de manera eficiente las lesiones malignas de las benignas: en presencia de hipoecogenicidad, microcalcificaciones y bordes irregulares existe una elevada prevalencia de CPT $(97,6 \%)$ y en ausencia de ellas ésta es muy baja (5,4\%), con likelihood ratios de 0,06 y 45 , respectivamente. En nuestro medio se asociaron a CPT: la presencia de microcalcificaciones, hipoecogenicidad, bordes irregulares, predominio sólido, adenopatía sospechosa, vascularización central e invasión local. Tras un análisis multivariado, sólo las tres primeras mantuvieron la significación y permitieron la confección de la escala descrita.

En relación a estas variables, publicaciones previas comunican una especificidad que fluctúa entre $85 \%$ y $95 \%$ para microcalcificaciones, $83 \%$ y $85 \%$ para bordes irregulares y $80 \%$ asociado a la vascularización de predominio central ${ }^{3,5,19,20}$. La

Tabla 2. Escala de puntaje predictiva para carcinoma papilar tiroideo (C PT)

\begin{tabular}{|lccc|}
\hline Puntaje & $\begin{array}{c}\text { Porcentaje de } \\
\text { carcinoma papilar }\end{array}$ & $\begin{array}{c}\text { Porcentaje de } \\
\text { lesiones benignas }\end{array}$ & $\begin{array}{c}\text { Likelihood } \\
\text { ratio }\end{array}$ \\
\hline 0 & 5,4 & 94,6 & 0,06 \\
$1-2$ & 14,3 & 85,7 & 0,15 \\
$3-5$ & 72,8 & 27,2 & 2,37 \\
6 & 97,6 & 2,4 & 45 \\
\hline
\end{tabular}

Borde irregular: 1 punto; Hipoecogenicidad: 2 puntos; Microcalcificaciones: 3 puntos. 
asociación de cualquiera de las anteriores con hipoecogenicidad en algunos trabajos ha permitido reconocer cerca de $90 \%$ de los $\mathrm{CPT}^{3,5,19,20}$, permitiendo así, a juicio de los autores, plantear la disminución del número de PAAF para el estudio de los nódulos tiroideos ${ }^{4}$. Uno de los problemas reportados en estos estudios radica en la limitada sensibilidad de estas características, lo que ha limitado su capacidad predictiva ${ }^{3}$. Aunque no mantuvieron la significación en la regresión logística, debemos reconocer la utilidad de variables como la presencia de adenopatía sospechosa e invasión local, que pese a tener una sensibilidad limitada para CPT, 26\% y 7,6\%, respectivamente, tuvieron una alta especificidad derivada de su aún menor representación en el grupo de nódulos benignos: 2,8\% la primera y $0 \%$ la última. Esto permite, en presencia de cualquiera de ellas, y aun en ausencia de las características que componen la escala propuesta, estimar un alto riesgo de $\mathrm{CPT}$, que debiera motivar el estudio dirigido con PAAF de estos nódulos ${ }^{2,3}$.

El cálculo de la razón de verosimilitud o likelihood ratio (LR), permite estimar la probabilidad postest de CPT en todo paciente sometido a ecografía tiroidea $^{18}$. Así, LR sobre 10 y bajo 0,1, como los obtenidos en este estudio, producen una significativa variación de la probabilidad pretest de una patología y, derivado de ello, generan cambios en el enfrentamiento o manejo de un paciente, que en este caso podrían, por ejemplo, sugerir el seguimiento ecográfico en ausencia de las 3 variables (LR 0,06), considerar la tiroidectomía total en presencia de las 3 variables (LR 45) 3,5,18, y realizar estudio con PAAF en aquellos nódulos con puntaje intermedio (1 a 5). Esto cobra aún más importancia si consideramos que $50 \%$ de los nódulos tiroideos incluidos en esta serie tuvo 0 ó 6 puntos en la evaluación ecográfica.

Otra de las ventajas de la escala generada es su composición por tres variables de fácil reconoci-

\section{REFERENCIAS}

1. Ezzat S, Sarti DA, Cain DR, Braunstein GD. Thyroid incidentalomas. Prevalence by palpation and ultrasonography. Arch Intern Med 1994; 154: 1838-40.

2. Hegedus L. The thyroid nodule. N Engl J Med 2004; 351: 1764-71. miento ecográfico y cómoda retención por el clínico, lo que posibilitaría su realización por equipos que no sean altamente sofisticados, aun cuando la buena calidad de una imagen obtenida con un equipo de última generación facilita la detección de las características descritas.

En relación al menor tamaño de los CPT respecto a los nódulos benignos, estimamos que esto deriva de una práctica recomendada por diversas guías clínicas y expertos, que sugieren el estudio con PAAF de aquellas lesiones menores a $1 \mathrm{~cm}$ en presencia de características ecográficas asociadas a mayor riesgo de $\mathrm{CPT}^{3,21}$. Lo anterior confiere aún más utilidad a la escala generada, pues ayudará a discriminar de manera eficiente en este grupo de pacientes, a quienes más se beneficien del estudio con PAAF.

La principal limitación de este trabajo es la utilización del estudio histológico obtenido por PAAF para el diagnóstico de CPT y no el de la pieza quirúrgica completa, que es el patrón de oro en esta patología. En el subgrupo de nuestros pacientes que tenían estudio con PAAF que concluía CPT y además se realizaron tiroidectomía total, existió concordancia en 98,6\% de los casos, cifra similar a la descrita en la literatura, que reporta sensibilidad y especificidad promedio de $85 \%$ y 95\%, respectivamente $3,18,22$. Considerando los puntos anteriores, esta escala se encuentra en proceso de validación por medio de su aplicación prospectiva en pacientes tiroidectomizados con CPT y patología tiroidea benigna, evaluados previamente con ecografía por medio de un protocolo que certifica con detalle la presencia o ausencia de cada una de las características descritas.

En conclusión, presentamos una escala que estima de manera clínicamente eficiente la probabilidad de CPT en pacientes con nódulos tiroideos, lo que en el futuro podría derivar en cambios significativos en el manejo que actualmente se hace de esta patología.

3. Gharib H, Pappini E. Thyroid nodules: clinical importance, assessment, and treatment. Endocrinol Metab Clin N Am 2007; 36: 707-35.

4. Fardella C, Jiménez M, González H, León A, Goñi I, Cruz F ET AL. Características de presentación del microcarcinoma papilar del tiroides. Experiencia retrospectiva de los últimos doce años. Rev Méd Chile 2005; 133: 1305-10. 
5. Papini E, Guglielmi R, Bianchini A, Crescenzi A, TACCOGNa S, NARDi F ET al. Risk of malignancy in nonpalpable thyroid nodules: predictive value of ultrasound and color-Doppler features. J Clin Endocrinol Metab 2002; 87: 1941-6.

6. Mandel SJ. Diagnostic use of ultrasonography in patients with nodular thyroid disease. Endocr Pract 2004; 10: 246-52.

7. Kim JY, Lee CH, Kim SY, JeOn WK, Kang JH, An SK et AL. Radiologic and pathologic findings of nonpalpable thyroid carcinomas detected by ultrasonography in a medical screening center. J Ultrasound Med 2008; 27: 215-23.

8. Rago T, Santini F, Scutari M, Pinchera A, Vitti P. Elastography: new developments in ultrasound for predicting malignancy in thyroid nodules. J Clin Endocrinol Metab 2007; 92: 2917-22.

9. Alexander EK, Marqusee E, Orcutt J, Benson CB, Frates MC, Doubilet PM et al. Thyroid nodule shape and prediction of malignancy. Thyroid 2004; 14: 953-8.

10. Cappelli C, Castellano M, Pirola i, Gandossi E, De Martino E ET al. Thyroid nodule shape suggests malignancy. Eur J Endocrinol 2006; 155: 27-31.

11. Tae HJ, Lim DJ, Baek KH, Park WC, Lee YS, Choi JE et AL. Diagnostic value of ultrasonography to distinguish between benign and malignant lesions in the management of thyroid nodules. Thyroid 2007; 17: 461-6.

12. Kim EK, Park CS, Chung WY, Oh KK, Kim DI, Lee JT et AL. New sonographic criteria for recommending fineneedle aspiration biopsy of nonpalpable solid nodules of the thyroid. Am J Roentgenol 2002; 178: 687-91.

13. Ito Y, Amino N, Yokozawa T, Ота H, Ohshita M, Murata N ET AL. Ultrasonographic evaluation of thyroid nodules in 900 patients: comparison among ultrasonographic, cytological, and histological findings. Thyroid 2007; 17: 1269-76.

14. Hoang JK, Lee WK, Lee M, Johnson D, Farrell S. US Features of thyroid malignancy: pearls and pitfalls. Radiographics 2007; 27: 847-60.

15. Frates MC, Benson CB, Charboneau JW, Cibas ES, Clark OH, Coleman BG ET al. Management of thyroid nodules detected at US: Society of Radiologists in Ultrasound consensus conference statement. Radiology 2005; 237: 794-800.

16. Shimura H, Haraguchi K, Hiejima Y, Fukunari N, Fujimoto Y, Katagiri M et al. Distinct diagnostic criteria for ultrasonographic examination of papillary thyroid carcinoma: a multicenter study. Thyroid 2005; 15: 251-8.

17. Leboulleux S, Girard E, Rose M, Travagli JP, Sabbah N, CAILLOU B ET AL. Ultrasound criteria of malignancy for cervical lymph nodes in patients followed up for differentiated thyroid cancer. J Clin Endocrinol Metab 2007; 92: 3590-4.

18. Guyatt G, Sackett D, Haynes B. Evaluating diagnostic tests. En: Haynes B, Sackett D, Guyatt G, Tugwell D. Clinical Epidemiology. How to do clinical practice research. Lippincott Williams \& Wilkins 2006; 273322.

19. PAPini E. The dilemma of non palpable thyroid nodules. J Endocrinol Invest 2003; 26: 3-4.

20. Frasoldati A, Valcavi R. Challenges in neck ultrasonography: lymphadenopathy and parathyroid glands. Endocr Pract 2004; 10: 261-8.

21. Campusano C, Bello F, González R, Lam J, Liberman C, Munizaga F ET al. Consenso de diagnóstico y manejo de los nódulos tiroideos no palpables. Rev Méd Chile 2004; 132: 1249-55.

22. Valenzuela L, Cifuentes L. Validez de estudios de tests diagnósticos. Rev Méd Chile 2008; 136: 401-4. 